\title{
Novel Fractional-Order Lagrangian to Describe Motion of Beam on Nanowire
}

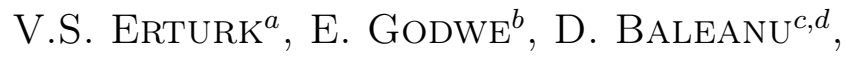 \\ P. $\operatorname{KUMAR}^{e}, J . \mathrm{ASAD}^{f, *}$ AND A. JAJARMI ${ }^{g}$ \\ ${ }^{a}$ Department of Mathematics, Faculty of Arts and Sciences, \\ Ondokuz Mayis University, 55139, Samsun, Turkey \\ ${ }^{b}$ Department of Physics, Faculty of Science, University of Maroua, P.O. Box 814, Cameroon \\ ${ }^{c}$ Department of Mathematics, Faculty of Arts and Sciences, \\ Cankaya University, 06530, Ankara, Turkey \\ ${ }^{d}$ Institute of Space Sciences, P.O. Box, MG-23, R 76900, Magurele, Bucharest, Romania \\ ${ }^{e}$ Department of Mathematics and Statistics, School of Basic and Applied Sciences, \\ Central University of Punjab, Bathinda, Punjab-151001, India \\ ${ }^{f}$ Department of Physics, Faculty of Applied Sciences, \\ Palestine Technical University, Tulkarm, Palestine \\ ${ }^{g}$ Department of Electrical Engineering, University of Bojnord, \\ P.O. Box, 94531-1339, Bojnord, Iran
}

Received: 12.07.2021 \& Accepted: 24.09.2021

Doi: $10.12693 /$ APhysPolA.140.265

*e-mail: j.asad@ptuk.edu.ps

\begin{abstract}
Our aim in this research is to investigate the motion of a beam on an internally bent nanowire by using the fractional calculus theory. To this end, we first formulate the classical Lagrangian which is followed by the classical Euler-Lagrange equation. Then, after introducing the generalized fractional Lagrangian, the fractional Euler-Lagrange equation is provided for the motion of the considered beam on the nanowire. An efficient numerical scheme is introduced for implementation and the simulation results are reported for different fractional-order values and various initial settings. These results indicate that the fractional responses approach the classical ones as the fractional order goes to unity. In addition, the fractional Euler-Lagrange equation provides a flexible model possessing more information than the classical description - the fact that leads to a considerably better evaluation of the hidden features of the real system under investigation.
\end{abstract}

topics: nanowire, fractional derivatives, fractional Lagrangian, simulation

\section{Introduction}

The idea of fractional calculus has already been proven by the precursors of common calculus. The authors of $[1,2]$ demonstrated that fractional calculus is required when researchers make scientific efforts to depict the complex nature of many real-life phenomena. It is incredible to realize that in previous years scientists, mainly engineers, have been ignorant of fractional calculus due to its non-real practical fields. Fortunately, the paradigm shift proceeded from the natural mathematical setup to applications in diverse fields. The fields of science, engineering, and mathematics have applied fractional calculus mostly in the last decades. The effects concern, for example, physics [3-5], control theory [6], and other fields. To be more specific, researchers have become increasingly interested in fractional calculus. With its use, for example, the fractional Lagrangian or Hamiltonian equations were derived for problems considered in $[7-10]$. To solve certain fractional differential equations, many numerical methods are applied such as the Grünwald-Letnikov approximation [1], and the variational iteration method [11].

In recent years, fractional calculus has also shown its importance for investigating physical and engineering systems that can be successfully described through differential equations. We refer the interested readers to [12-17] and references therein, where useful methods of great importance were presented. In [18], the authors simulated the Lagrangian mechanics of fractional order, in which the Hamilton-Jacobi non-classical type partial differential equation (PDE) and Taylor's series of nondifferentiable functions were discussed. In [19], the fractional-order Euler-Lagrange equations and dynamics of the Hamiltonian equations were introduced. A numerical technique based on noninteger order Lagrange polynomials for simulating 
a class of non-classical type differential equations was given in [20]. As an example, the multistep reduced differential transform method (DTM) was used to solve one-dimensional fractional heat equations with time fractional derivatives, and the Klein-Gordon equation. The results show that the method is a reliable technique that can be used to handle linear and nonlinear fractional partial differential equations $[12,13]$.

Also, the multistep generalized differential transform method was used to investigate both the Rabinovich-Fabrikant model involving the Caputo fractional derivative subjected to appropriate initial conditions and to construct a novel robust algorithm in finding numerical approximate solutions to the following model of the time-space fractional Fokker-Planck equation [15, 16]. In [21], the generalized Birkhoffian systems in the sense of the classical and combined Caputo fractionalderivatives were discussed while a fruitful theory on fractional-order differential equations was given in [2]. Further, in [22], the authors analyzed a numerical study on the constitutive characterization of thermoplastic materials submitted to finite strain.

The motion area often takes circular, conical, or parabolic shapes. Consequently, when describing the beam, bedbug, head, dust, etc. behaviors, one can perform an analysis similar to the particle analysis. In general, scientists and engineers concentrate on problems and tools that help them carry out their commands. Therefore, in this century, scientists have got interested in the pre-existing theory of random transformation. We naturally believe that such a great interest is just a fractal aspect of the world. Early in the 21st century, physicists have learned, basing on their knowledge and scientific research, how to apply inescapable equations on a complex structure/area.

It is worth mentioning that in fact several physical problems are solved by defining fractional operators. In this case, even if a mathematical object is well defined, the Caputo definition of the fractional derivative is required and applied to obtain a better solution to these problems. Similarly, to motivate a beam on the nanowire's concept, an example of $2 N$ nonlinear algebraic equations is elaborated. Thus, the desire to explore, to create a new model for the fractional integral becomes our particular interest. Nowadays, we are motivated by the consideration of pure mathematicians to generalize simple power potentials and extend them to cover a whole host of more information. That is why real-world systems (phenomena) lead to several interesting definitions of fractional calculus. Expanding the field of fractional calculus is one of the motivations.

In this work, we are interested in a beam moving on an internally bent nanowire with a more realistic shape. In order to analyze the motion of particles on these surfaces, analytical and numerical solutions may prove very useful. Therefore, it is quite correct to think that the new component of non-locality in the proposed system is revealed thanks to the numerical results of the fractional Euler-Lagrange equation (FELE) for the motion of a beam on a nanowire. As far as the mathematical and practical points of view are concerned, it will be recognized that the FELEs as well as their numerical simulations possess a lot of information, giving a better evaluation as compared to the corresponding integer-order ones.

The current paper is organized as follows. First, preliminaries concerning the basic explanations in fractional calculus are briefly presented in Sect. 2 . Next, we describe the Euler-Lagrange equation of motion in both classical and fractional frameworks in Sect. 3. Further, we carry out in detail the numerical method studied for a beam on a nanowire in Sect. 4 and we provide some numerical simulations in Sect. 5. Finally, Sect. 6 is devoted to the conclusion.

\section{Preliminaries}

Below, a brief explanation of the main meanings related to derivatives of fractional-order in the Caputo sense as well as their corresponding integrals can be found. Let $x(\xi)$ be a function in the region $(d, b)$ and belong to $\mathbb{R}$.

The left Caputo fractional derivative of order $\beta$ is defined as

${ }_{d}^{\mathrm{C}} D_{\xi}^{\beta} x(\xi)=\frac{1}{\Gamma(m-\beta)} \int_{d}^{\xi} \mathrm{d} \vartheta(\xi-\vartheta)^{m-\beta-1} x^{(m)}(\vartheta)$.

The right Caputo fractional derivative, with the same order as (1), is defined as

${ }_{\xi}^{\mathrm{C}} D_{b}^{\beta} x(\xi)=\frac{(-1)^{m}}{\Gamma(m-\beta)} \int_{\xi}^{b} \mathrm{~d} \vartheta(\vartheta-\xi)^{m-\beta-1} x^{(m)}(\vartheta)$,

in which $\Gamma(\cdot)$ is the gamma function, and $n$ is the integer element such that $m-1<\beta<m$. The corresponding fractional left and right integrals are, respectively, described as

$$
{ }_{d} I_{\xi}^{\beta} x(\xi)=\frac{1}{\Gamma(\beta)} \int_{d}^{\xi} \mathrm{d} \vartheta(\xi-\vartheta)^{\beta-1} x(\vartheta)
$$

and

$$
I_{b}^{\beta} x(\xi)=\frac{1}{\Gamma(\beta)} \int_{\xi}^{b} \mathrm{~d} \vartheta(\vartheta-\xi)^{\beta-1} x(\vartheta) .
$$

Note that the Caputo derivative coincides with the ordinary differentiation when $\beta$ is the integer, i.e.,

$$
\begin{aligned}
\quad{ }_{d}^{\mathrm{C}} D_{\xi}^{\beta} x(\xi) & =x^{(m)}(\xi) \\
\text { and } & \\
{ }_{\xi}^{\mathrm{C}} D_{b}^{\beta} x(\xi) & =(-1)^{m} x^{(m)}(\xi) .
\end{aligned}
$$

Further information can be found in [1]. 


\section{System description}

As can be seen from the literature cited in the previous sections, some functions have already been proposed and much work has been performed on them. Now, our goal is to define a new function that will give this topic a new aspect.

In this section, the nonlinear system of a beam bent into a more realistic potential of the shape $z$ is considered. Its form is $z=\frac{A}{2 !} x^{2}-\frac{B}{4 !} x^{4}+\frac{C}{6 !} x^{6}$, where $x$ is the displacement, $A$ is the natural frequency, $B$ are the coefficients of the quartic, and $C$ are the coefficients of the sextic nonlinearities. Remarkably, this type of potential can exhibit a variety of configurations like monostable, bistable, and tristable non-catastrophe ones depending on the values of physical parameters $A, B$, and $C$. They are equivalent to the cases of beams, soft or devised structures. Here, we focus our attention on the beams in order to find the interest and the potential dynamics provided by additive 4th- and 6th-order polynomials considering their symmetry. Many works have been carried out on this kind of potentials [23-25]. We suppose that the axis is perpendicular to the gravitational field of the earth $g$. A beam that has a mass $m$ moves along the nanowire of a given roughness. The expressions of the kinetic and potential energy of the beam are given, respectively, as

$$
\begin{gathered}
K=\frac{m}{2}\left(\dot{x}^{2}+\dot{z}^{2}\right)=\frac{m}{2} \dot{x}^{2}\left(1+A^{2} x^{2}+\frac{B^{2}}{36} x^{6}\right. \\
\left.+\frac{C^{2}}{14400} x^{10}-\frac{A B}{3} x^{4}+\frac{A C}{60} x^{6}-\frac{B C}{360} x^{8}\right)
\end{gathered}
$$

and

$$
V=m g z=m g\left(\frac{A}{2} x^{2}-\frac{B}{24} x^{4}+\frac{C}{720} x^{6}\right) .
$$

Furthermore, the conventional Lagrangian is written as

$$
\begin{aligned}
L & =K-V=\frac{1}{2} m \dot{x}^{2}\left(1+A^{2} x^{2}+\frac{B^{2}}{36} x^{6}\right. \\
& \left.+\frac{C^{2}}{14400} x^{10}-\frac{A B}{3} x^{4}+\frac{A C}{60} x^{6}-\frac{B C}{360} x^{8}\right) \\
& -\frac{m g}{2}\left(A x^{2}-\frac{B}{12} x^{4}+\frac{C}{360} x^{6}\right) .
\end{aligned}
$$

Based on (9), we can easily compute the classical Euler-Lagrange equation (CELE), i.e.,

$$
\frac{\partial L}{\partial x}-\frac{\mathrm{d}}{\mathrm{d} t} \frac{\partial L}{\partial \dot{x}}=0
$$

Considering (9) and (10), the CELE is presented by

$$
\begin{aligned}
m \ddot{x} & \left(1+A^{2} x^{2}-\frac{A B}{3} x^{4}+\frac{B^{2}}{36} x^{6}+\frac{A C}{60} x^{6}\right. \\
- & \left.\frac{B C}{360} x^{8}+\frac{C^{2}}{14400} x^{10}\right) \\
+ & m \dot{x}^{2}\left(A^{2} x-\frac{2 A B}{3} x^{3}+\frac{B^{2}}{12} x^{5}+\frac{A C}{20} x^{5}\right. \\
& \left.-\frac{B C}{90} x^{7}+\frac{C^{2}}{2880} x^{9}\right) \\
& +m g\left(A x-\frac{B}{6} x^{3}+\frac{C}{120} x^{5}\right)=0 .
\end{aligned}
$$

Furthermore, we explore the fractional picture of the standard Lagrangian (9), which shows the novel elements of the physical setup under attention. The main motivation to do this replacement is to check how the given dynamics will behave under the $\mathrm{Ca}$ puto derivative versus the time-variable $t$. This replacement allows the inclusion of variations in the given dynamics which can be made by the non-local properties of fractional derivatives. To this aim, our first step is generalizing (9) and rewriting it in the fractional framework

$$
\begin{aligned}
L^{\mathrm{F}} & =\frac{m\left({ }_{a}^{\mathrm{C}} D_{t}^{\alpha} x\right)^{2}}{2}\left(1+A^{2} x^{2}+\frac{B^{2}}{36} x^{6}+\frac{C^{2}}{14400} x^{10}\right. \\
& \left.-\frac{A B}{3} x^{4}+\frac{A C}{60} x^{6}-\frac{B C}{360} x^{8}\right) \\
& -\frac{m g}{2}\left(A x^{2}-\frac{B}{12} x^{4}+\frac{C}{360} x^{6}\right) .
\end{aligned}
$$

Then, the FELE of motion is obtained from

$$
\frac{\partial L^{\mathrm{F}}}{\partial x}+{ }_{t}^{\mathrm{C}} D_{b}^{\alpha} \frac{\partial L^{\mathrm{F}}}{\partial_{a}^{\mathrm{C}} D_{t}^{\alpha} x}+{ }_{a}^{\mathrm{C}} D_{t}^{\beta} \frac{\partial L^{\mathrm{F}}}{\partial_{t}^{\mathrm{C}} D_{b}^{\alpha} x}=0 .
$$

Here, parameters $a$ and $b$ are the terminals to indicate the left and right approaches of the given $\mathrm{Ca}$ puto derivative on the proposed time-span. As a result of using (11) and (12), one leads to the form of (13) as follows

$$
\begin{gathered}
m\left({ }_{a}^{\mathrm{C}} D_{t}^{\alpha} x\right)^{2}\left[A^{2} x+\left(\frac{B^{2}}{12}+\frac{A C}{20}\right) x^{5}+\frac{C^{2}}{2880} x^{9}-\frac{2 A B}{3} x^{3}-\frac{B C}{90} x^{7}\right]-m g\left(A x-\frac{B}{6} x^{3}+\frac{C}{120} x^{5}\right) \\
+m\left({ }_{t}^{\mathrm{C}} D_{b}^{\alpha}\right)\left({ }_{a}^{\mathrm{C}} D_{t}^{\alpha} x\right)\left(1+A^{2} x^{2}+\frac{B^{2}}{36} x^{6}+\frac{C^{2}}{14400} x^{10}-\frac{A B}{3} x^{4}+\frac{A C}{60} x^{6}-\frac{B C}{360} x^{8}\right)=0 .
\end{gathered}
$$

It should be noted that as $\alpha \rightarrow 1$, the FELE (14) becomes like the CELE (11). In the following part, our goal is to numerically treat (14) for some fractional values and initial settings.

\section{Numerical technique}

The development of an approximation scheme for the fractional Euler-Lagrange system (14) primarily requires its reformulation. 
Let us expatiate on the new variable $\varpi(t)$ as $\varpi(t):={ }_{a}^{C} D_{t}^{\alpha} x(t)$. Consequently, (14) is converted to

${ }_{a}^{\mathrm{C}} D_{t}^{\alpha} x(t)=\varpi(t)$

$$
\begin{aligned}
&{ }_{t}^{\mathrm{C}} D_{b}^{\alpha} \varpi(t) {\left[1+A^{2} x^{2}(t)-\frac{A B}{3} x^{4}(t)+\left(\frac{B^{2}}{36}+\frac{A C}{60}\right) x^{6}(t)-\frac{B C}{360} x^{8}(t)+\frac{C^{2}}{14400} x^{10}(t)\right] } \\
&+\varpi^{2}(t)\left[A^{2} x(t)-\frac{2 A B}{3} x^{3}(t) x^{5}(t)+\left(\frac{B^{2}}{12}+\frac{A C}{20}\right) x^{5}(t)-\frac{B C}{90} x^{7}(t)+\frac{C^{2}}{2880} x^{9}(t)\right]= \\
& g A x(t)-\frac{g B}{6} x^{3}(t)+\frac{g C}{120} x^{5}(t)=0 .
\end{aligned}
$$

The results given in (15) and (16) agree with the Volterra integral equations, respectively, as

$$
x(t)=x(a)+\int_{a}^{t} \mathrm{~d} \tau(t-\tau)^{\alpha-1} \varpi(\tau),
$$

and

$$
\begin{aligned}
& \varpi(t)\left[1+A^{2} x^{2}(t)-\frac{A B}{3} x^{4}(t)+\left(\frac{B^{2}}{36}+\frac{A C}{60}\right) x^{6}(t)-\frac{B C}{360} x^{8}(t)+\frac{C^{2}}{14400} x^{10}(t)\right]= \\
& \varpi(b)\left[1+A^{2} x^{2}(b)-\frac{A B}{3} x^{4}(b)+\left(\frac{B^{2}}{36}+\frac{A C}{60}\right) x^{6}(b)^{6}-\frac{B C}{360} x^{8}(b)+\frac{C^{2}}{14400} x^{10}(b)\right] \\
& -\frac{1}{\Gamma(\alpha)} \int_{t}^{b} \mathrm{~d} \tau\left\{\varpi(\tau)^{2}\left[A^{2} x(\tau)-\frac{2 A B}{3} x^{3}(\tau)+\left(\frac{B^{2}}{12}+\frac{A C}{20} x^{5}(\tau)-\frac{B C}{90} x^{7}(\tau)+\frac{C^{2}}{2880} x^{9}(\tau)\right)\right]\right. \\
& \left.-g A x(\tau)+\frac{g B}{6} x^{3}(\tau)-\frac{g C}{120} x^{5}(\tau)\right\} .
\end{aligned}
$$

Now, we take into account a uniform network on $[a, b])$ and mark the nodes $0,1, \ldots, M$, where $M$ is the arbitrary natural number, and $h_{M}=(b-a) / M$ is the time increment. We indicate $\left(x_{i}, \varpi_{i}\right)$ as the numerical approximation of $\left(x\left(t_{i}\right), \varpi\left(t_{i}\right)\right)$, where $t_{i}=a+i h_{M}$ is the time at node $i$ for $0 \leqslant i \leqslant M$. Apart from this, we approximate $\varpi(t)$ and $x(t)$ intercepted within two sequential temporary nodes linearly. Basing on the above preliminaries and in accordance with Hasan et al. [26], one can reduce (15) and (16) to

$$
x\left(t_{i}\right)=x_{0}+\sum_{j=0}^{i} a_{i j} \varpi\left(t_{j}\right),
$$

where, for $i=1, \ldots$, the coefficients $a_{i j}$ are defined as

$$
a_{i j}=d_{1} \begin{cases}(i-1)^{\beta}-i^{\beta}+\beta i^{\alpha}, & \text { if } j=0 \\ (k+1)^{\beta}-2 k^{\beta}+(k-1)^{\beta}, & \text { if } 1 \leq j \leq i-1, \\ 1, & \text { if } j=i\end{cases}
$$

Here, $d_{1}=\frac{h^{\alpha}}{\Gamma(\alpha+2)}, \beta=\alpha+2$, and $k=i-j$. From the same viewpoint, the value of the following function

$$
\varpi(t)\left[1+A^{2} x^{2}(t)-\frac{A B}{3} x^{4}(t)+\left(\frac{B^{2}}{36}+\frac{A C}{60}\right) x^{6}(t)-\frac{B C}{360} x^{8}(t)+\frac{C^{2}}{14400} x^{10}(t)\right]
$$

at the node $i$ will be

$$
\begin{aligned}
& \varpi\left(t_{i}\right)\left[1+A^{2} x^{2}\left(t_{i}\right)-\frac{A B}{3} x^{4}\left(t_{i}\right)+\left(\frac{B^{2}}{36}+\frac{A C}{60}\right) x^{6}\left(t_{i}\right)-\frac{B C}{360} x^{8}\left(t_{i}\right)+\frac{C^{2}}{14400} x^{10}\left(t_{i}\right)\right]= \\
& \sum_{j=i}^{n} b_{i j}\left\{\varpi\left(t_{j}\right)^{2}\left[A^{2} x\left(t_{j}\right)-\frac{2 A B}{3} x^{3}\left(t_{j}\right)+\left(\frac{B^{2}}{12}+\frac{A C}{20}\right) x^{5}\left(t_{j}\right)-\frac{B C}{90} x^{7}\left(t_{j}\right)+\frac{C^{2}}{2880} x^{9}\left(t_{j}\right)\right)\right] \\
& \left.-g A x\left(t_{j}\right)+\frac{g B}{6} x^{3}\left(t_{j}\right)-\frac{g C}{120} x^{5}\left(t_{j}\right)\right\}
\end{aligned}
$$


where

$$
a_{i j}=d_{1} \begin{cases}(M-1)^{\beta}-M^{\beta}+\beta M^{\alpha}, & \text { if } j=N \\ (k+1)^{\beta}-2 k^{\beta}+(k-1)^{\beta}, & \text { if } i+1 \leq j \leq N-1, \\ 1, & \text { if } j=i\end{cases}
$$

Here, $M=N-i$ and $k=j-i$. Note that (19) and (22) include $2 N$ unknowns as well as $2 N$ nonlinear algebraic equations, so a well-known root finding technique, like the Newton method, can be used to solve them.

\section{Simulation results}

In this part, we put particular emphasis on the numerical results for the above-targeted problem. For different values of $h$ and $\alpha$, the solution is found. Below, we take $m=2$ and $g=9.81$. Moreover, we take the following condition for $\varpi(t)$ as $\varpi(0)=0$. In Figs. 1-4, the simulations of $x(t)$ and $z(t)=\frac{A}{2} x(t)^{2}-\frac{B}{24} x(t)^{4}+\frac{C}{720} x(t)^{6}$ are given for $\alpha=0.8,0.85,0.9,0.95,1$ and different values of $x(0)$. In these figures, we also give the answer of (11) in addition to some responses of (14) for $0<\alpha \leqslant 1$. Figures $1-4$ verify that the numerical result of the fractional Euler-Lagrange system (11) approaches the classical one as $\alpha$ tends to 1 . Thus, by looking for the effect of FELEs, they provide more flexible models, which leads to a considerably better evaluation of the hidden features of real-world phenomena. Also, we observed that the values of $A, B, C$ and $x(0)$ affect the dynamics of the proposed model. In Figs. 5 and 6 , the simulations of $\varpi(t)$ are shown for $\alpha=0.8,0.85$, $0.9,0.95$, and 1 . Here, we received some uniform changes in the peak of the oscillations when we changed the fractional-order values. Furthermore, the behavior of $x(t)$ as compared to $\varpi(t)$ is displayed in Figs. 7 and 8 . In these figures, when we changed the fractional-order values, then we observed some stretching in the given integer-order ring shape which justifies that the fractional-order dynamics is slightly different to the classical one. Figure 9 shows (a) $x(t)$ and (b) $\varpi(t)$ for $\alpha=0.8$ and $h=0.1,0.05$, and 0.025 . We realize that the solution converges when $h$ decreases. It is surely true that $h$ is less than 0.025 . This likely makes the scheme to be numerically constant, that is to say, stable. The same arguments can be given for the other values of $\alpha$. From the given practical simulations, we observed that the fractionalorder system performed very well to study the given phenomena. The main feature of the given fractional model is that we can simulate the proposed dynamics very clearly for the classical as well as non-classical sense.

Results of the calculation of (a) $x(t)$ and (b) $z(t)=\frac{A}{2} x^{2}(t)-\frac{B}{24} x^{4}(t)+\frac{C}{720} x^{6}(t)$ for $A=1.0$, $B=0.0706, C=0.0034, x(0) \stackrel{2=}{=} 0.1$, and different orders of $\alpha$ are displayed in Fig. 1.

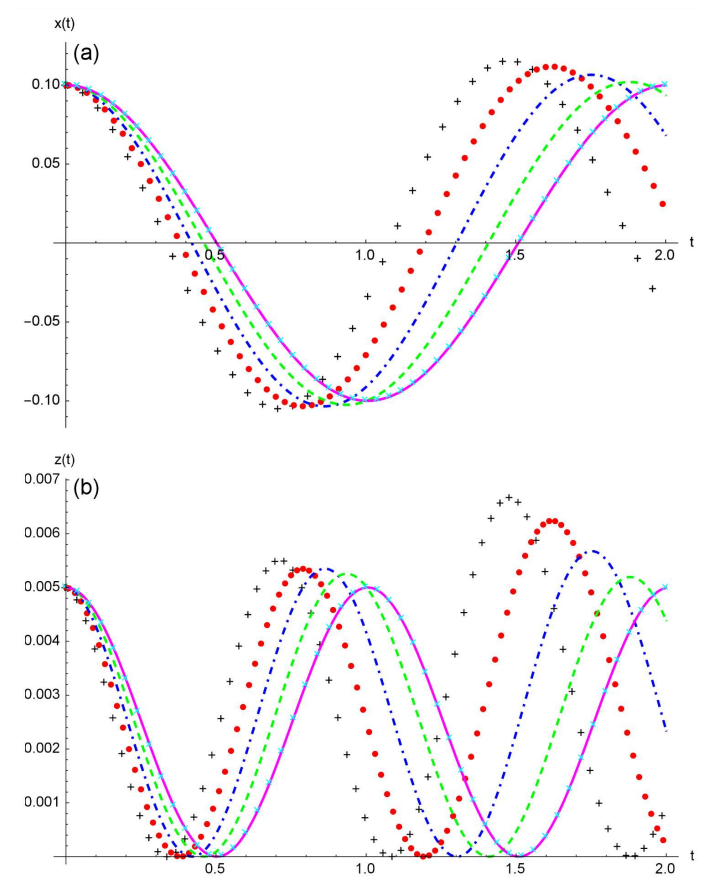

Fig. 1. Calculation results of $\alpha=0.8(+++)$, $\alpha=0.85(\cdots), \alpha=0.9(-\cdot-), \alpha=0.95(---)$, $\alpha=1(-)$, classic $(\times \times \times)$.

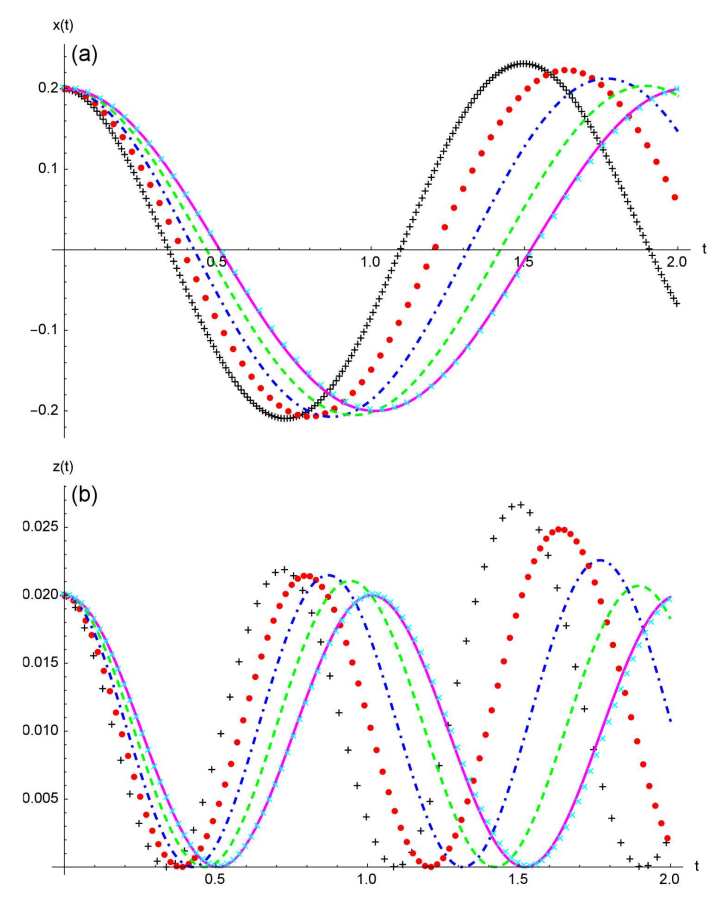

Fig. 2. Behavior of $\alpha=0.8 \quad(+++), \quad \alpha=0.85$ $(\cdots), \alpha=0.9(-\cdot-), \alpha=0.95(---), \alpha=1(-)$, classic $(\times \times \times)$. 

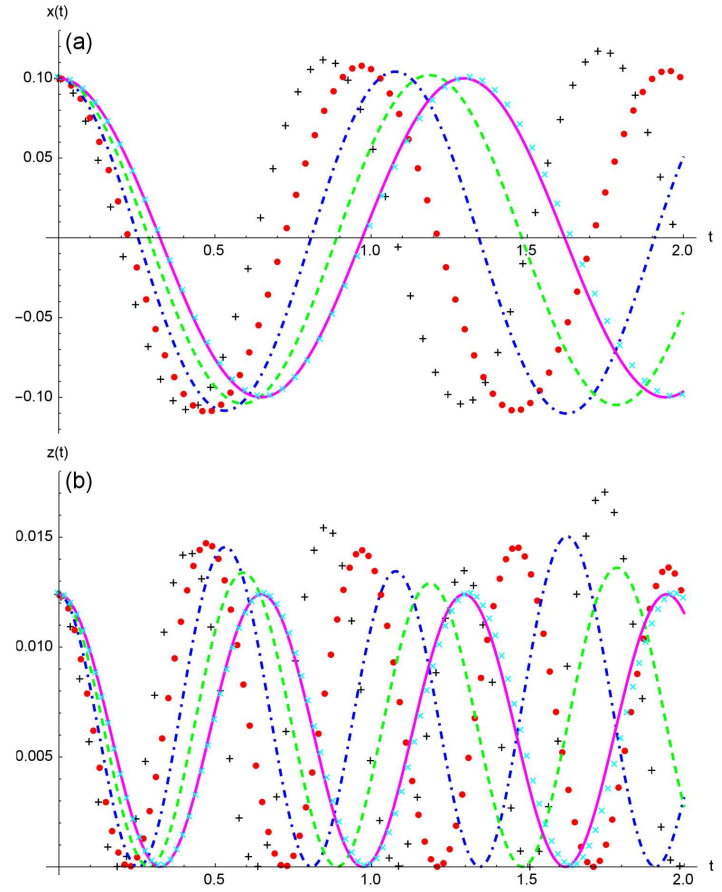

Fig. 3. Calculation results of $\alpha=0.8(+++)$, $\alpha=0.85(\cdots), \alpha=0.9(-\cdot-), \alpha=0.95(---)$, $\alpha=1(-)$, classic $(\times \times \times)$.
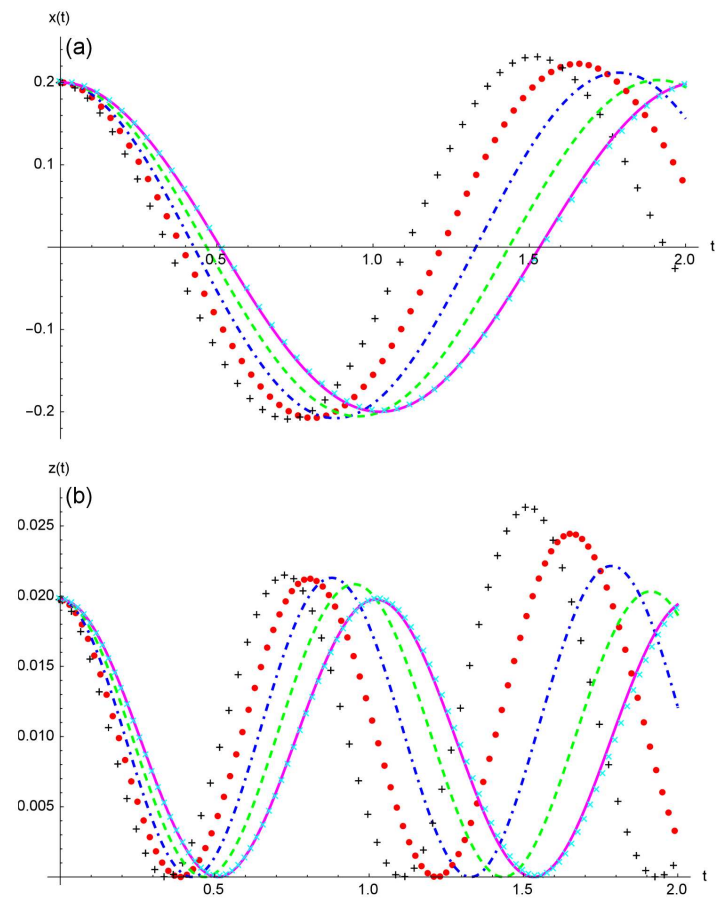

Fig. 4. Behavior of $\alpha=0.8(+++), \quad \alpha=0.85$ $(\cdots), \alpha=0.9(-\cdot-), \alpha=0.95(---), \alpha=1(-)$, classic $(\times \times \times)$.

Plots of (a) $x(t)$ and (b) $z(t)=\frac{A}{2} x^{2}(t)-$ $\frac{B}{24} x^{4}(t)+\frac{C}{720} x^{6}(t)$ for $A=1.0, B=0.0706$, $C=0.0034, x(0)=0.2$, and different orders of $\alpha$ are shown in Fig. 2.
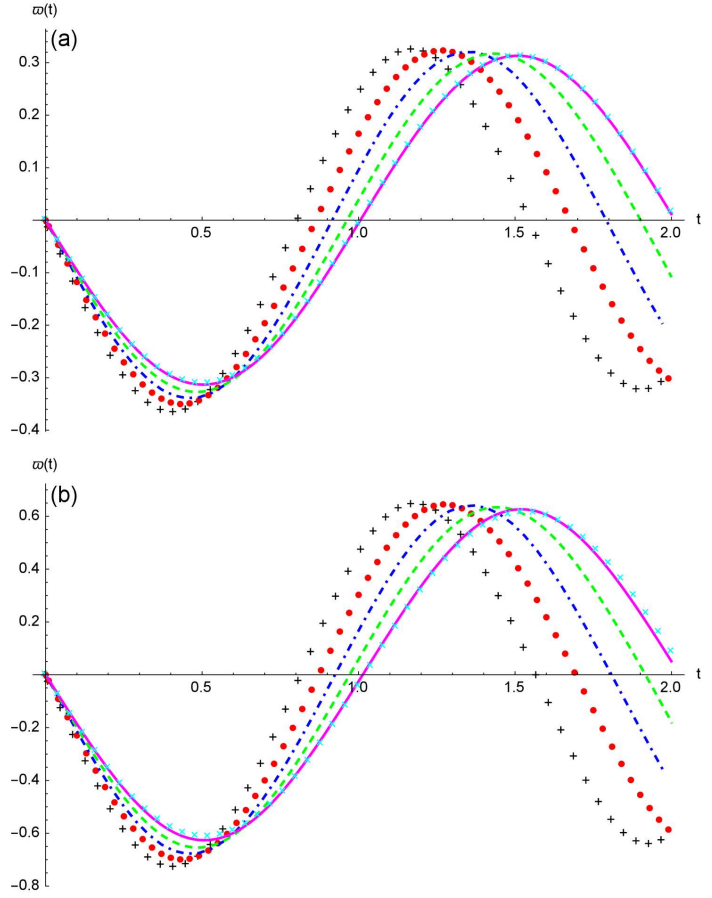

Fig. 5. Calculation results of $\alpha=0.8(+++)$, $\alpha=0.85(\cdots), \alpha=0.9(-\cdot-), \alpha=0.95(---)$, $\alpha=1(-)$, classic $(\times \times \times)$.
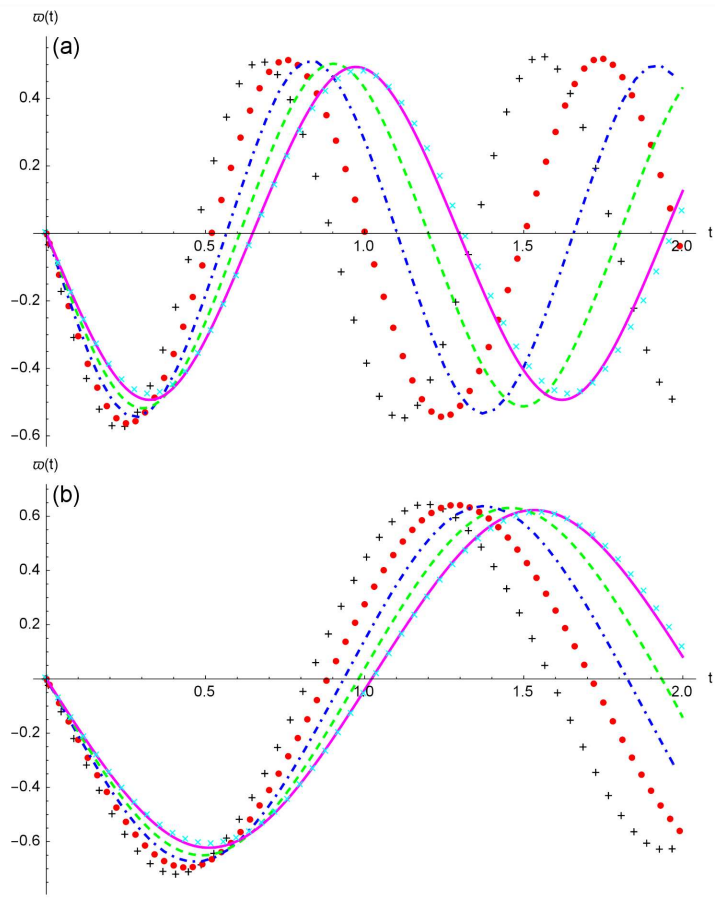

Fig. 6. Behavior of $\alpha=0.8 \quad(+++), \quad \alpha=0.85$ $(\cdots), \alpha=0.9(-\cdot-), \alpha=0.95(---), \alpha=1(-)$, classic $(\times \times \times)$.

Results of the calculation of (a) $x(t)$ and (b) $z(t)=\frac{A}{2} x^{2}(t)-\frac{B}{24} x^{4}(t)+\frac{C}{720} x^{6}(t)$ for $A=2.5$, $B=24, C=120, x(0)=0.1$, and different orders of $\alpha$, are presented in Fig. 3 . 

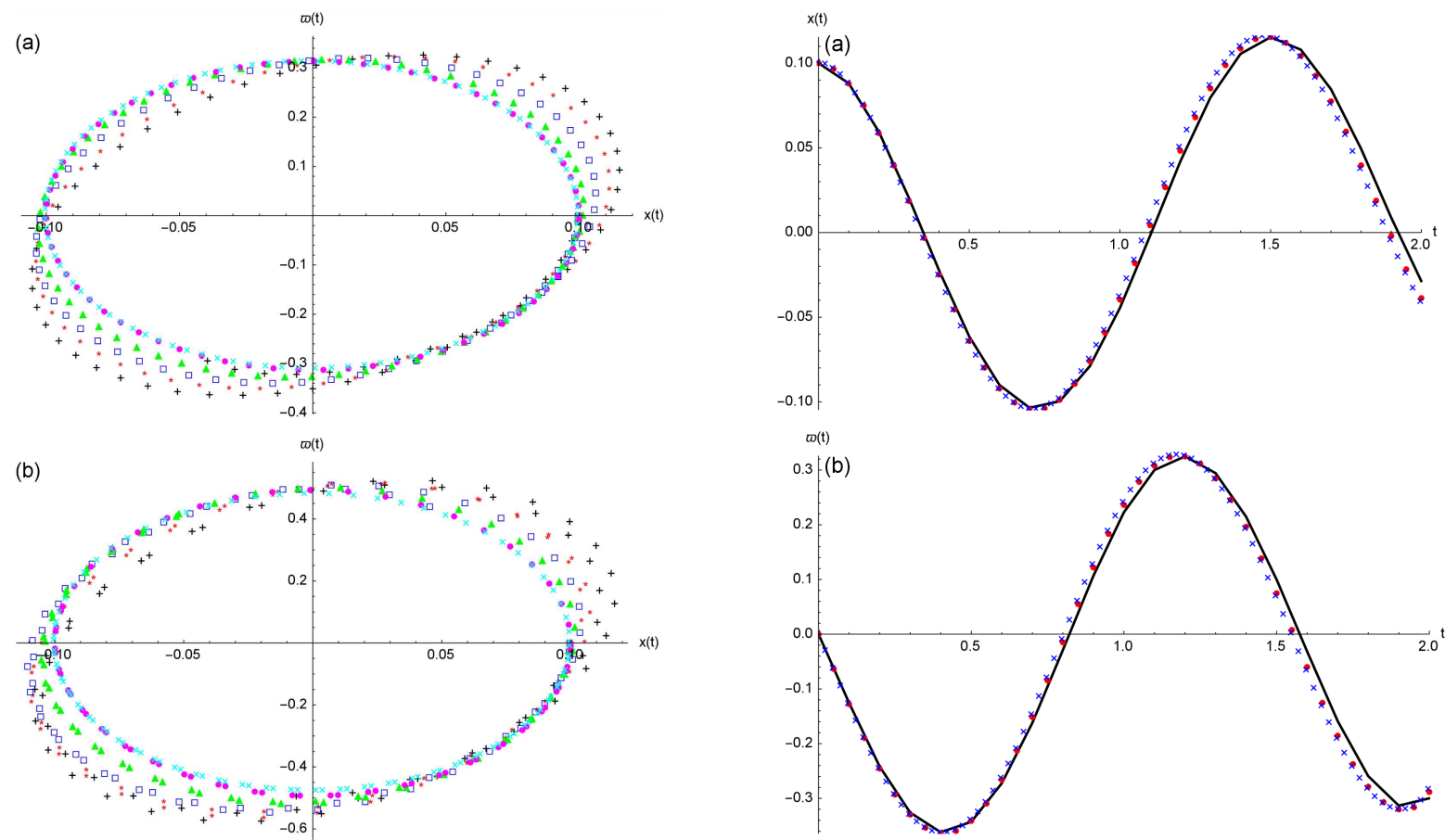

Fig. 7. Calculation results obtained for $\alpha=0.8$ $(+++), \alpha=0.85(* * *), \alpha=0.9$ (ㅁ口), $\alpha=0.95$ $(\boldsymbol{\Lambda} \mathbf{\Lambda} \mathbf{\Delta}), \alpha=1(\cdots)$, classic $(\times \times \times)$.

Fig. 9. Convergence of the variables $x(t)$ and $\varpi(t)$ for $\alpha=0.8: h=0.1(-), h=0.05(\cdots), h=0.025$ $(\times \times \times)$.
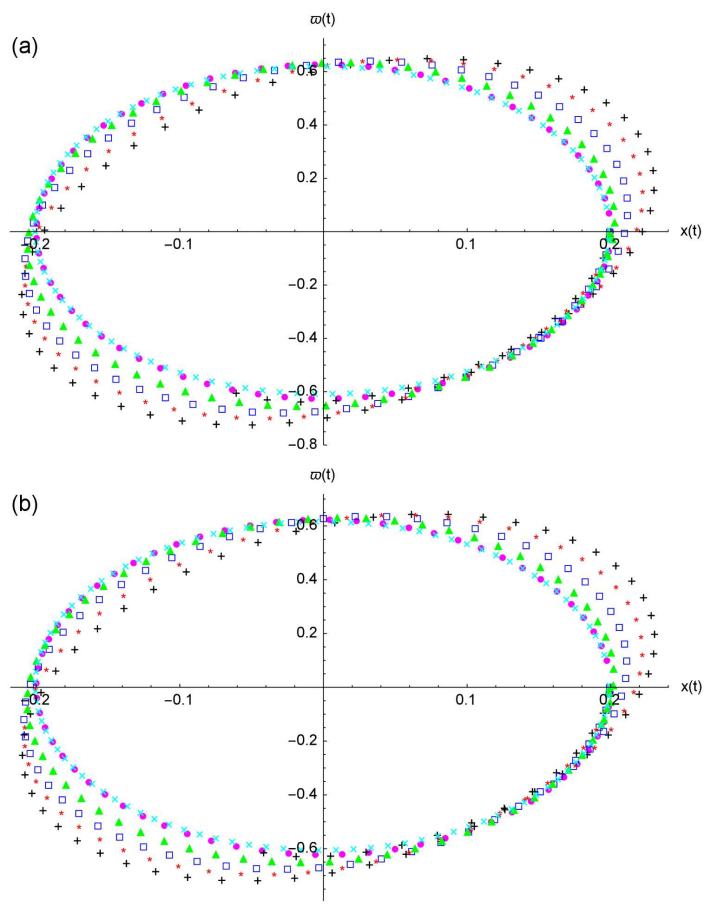

Results of the calculation of $\varpi(t)$ for $A=1.0$, $B=0.0706, \quad C=0.0034, \quad$ (a) $x(0)=0.1$, (b) $x(0)=0.2$ and different orders of $\alpha$ are charted in Fig. 5.

Plots of $\varpi(t)$ for (a) $A=2.5, B=24, C=120$, and $x(0)=0.1$, (b) $A=1.0, B=3.6, C=6$, and $x(0)=0.2$, and different orders of $\alpha$ are displayed in Fig. 6.

Results of the calculation of $x(t)$ versus $\varpi(t)$ for $A=1.0, B=0.0706, C=0.0034$, (a) $x(0)=0.1$, (b) $x(0)=0.2$ and different orders of $\alpha$ are displayed in Fig. 7 .

Behaviors of $x(t)$ versus $\varpi(t)$ for (a) $A=2.5$, $B=24, C=120$, and $x(0)=0.1$, (b) $A=1.0$, $B=3.6, C=6$, and $x(0)=0.2$ and different orders of $\alpha$ are displayed in Fig. 8 .

\section{Conclusion}

This paper employed the concept of the fractional Lagrangian approach to describe the motion of a beam on a nanowire. First, the classical Lagrangian was formulated, and the CELE was derived. Then, the generalized fractional Lagrangian was introduced, which led to the FELE for the motion of the beam on the nanowire. The efficient approximation method implemented the new model, and some simulation results described the associated dynamical behaviors. According to the obtained results, the fractional simulations tend to the classical responses as the fractional order goes to unity. In addition, the FELE enables us to 
make a considerably better evaluation of the hidden aspects of the real-world system under study. Consequently, it can be concluded that the FELE studied in this paper is new, and its numerical simulations presented possess more information as compared to the corresponding CELE. The advantage to use this fractional model is that we can study the given dynamics very clearly for the integer as well as non-integer sense.

\section{References}

[1] A.A. Kilbas, H.M. Srivastava, J.J. Trujillo, Theory and Applications of Fractional Differential Equations, Elsevier, Amsterdam 2006.

[2] I. Podlubny, Fractional Differential Equations, Vol. 198 of Mathematics in Science and Engineering, Academic Press, San Diego 1999.

[3] D. Baleanu, S.S. Sajjadi, A. Jajarmi, O. Defterli, J.H. Asad, Roman. Rep. Phys. 73, 105 (2021).

[4] J.H. Asad, D. Baleanu, B. Ghanbari, A. Jajarmi, H. Mohammadi Pirouz, CMESComput. Model. Eng. Sci. 124, 953 (2020).

[5] D. Baleanu, A. Jajarmi, J.H. Asad, T. Blaszczyk, Acta Phys. Pol. A 131, 1561 (2017).

[6] A. Jajarmi, D. Baleanu, Asian J. Contr. 23, 1062 (2021).

[7] F. Riewe, Phys. Rev. E 53, 1890 (1996).

[8] F. Riewe, Phys. Rev. E 55, 3581 (1997).

[9] O.P. Agrawal, J. Math. Anal. Appl. 272, 368 (2002).

[10] O.P. Agrawal, J. Vibrat. Acoust. 126, 561 (2004).

[11] S. Momani, Z. Odibat, A. Alawneh, $N u$ mer. Meth. Part. Diff. Eq. J. 24, 262 (2008).
[12] M. Al-Smadi, A. Freihat, H. Khalil, S. Momani, R.A. Khan, Int. J. Computat. Meth. 14, 1750029 (2017).

[13] S. Momani, O. Abu Arqoub, A. Freihat, M. Al-Smadi, Appl. Computat. Math. 15, 319 (2016).

[14] K. Moaddy, A. Freihat, M. Al-Smadi, Eman Abuteen, I. Hashim, Soft Comput. 22, 773 (2018).

[15] M. Al-Smadi, A. Freihat, M. Abu Hammad, S. Momani, O. Abu Arqub, J. Computat. Theor. Nanosci. 13, 7793 (2016).

[16] M. Al-Smadi, A. Freihat, O. Abu Arqoub, N. Shawagfeh, J. Computat. Anal. Appl. 19, 713 (2015).

[17] S. Momani, A. Freihat, M. Al-Smadi, Abstr. Appl. Anal. 2014, 276279 (2014).

[18] G. Jumarie, Chaos Solitons Fractals 32 , 969 (2007).

[19] M.A.E. Herzallah, D. Baleanu, Nonlin. Dyn. 58, 385 (2009).

[20] S. Sabermahani, Y. Ordokhani, S.A. Yousefi, Computat. Appl. Math. 37, 3846 (2018).

[21] Z. Ying, Y. Zhang, Acta Mech. 231, 3017 (2020).

[22] B.F. Luiz, J.M. Vassoler, Int. J. Solids Struct. 206, 456 (2020).

[23] R. Tchoukuegno, P. Woafo, Physica D 167, 86 (2002).

[24] Z.J. Jing, Z.Y. Yang, T. Jiang, Chaos Solitons Fractals 27, 722 (2006).

[25] Z.K. Sun, W. Xu, X.L. Yang, Chaos Solitons Fractals 27, 778 (2006).

[26] M.M. Hasan, X.W. Tangpong, O.P. Agrawal, J. Vibrat. Contr. 18, 1506 (2011). 